linie mehr Durchsetzungskraft zu verleihen, werde nicht durch die Brille der Nationalstaaten und liebgewonnener oder traditioneller Eigenheiten geschaut, sondern durch die EU-Binnenmarktbrille. Die Dienstleistungsrichtlinie aus dem Jahr 2006 hat den Abbau von bürokratischen Hindernissen und zwischenstaatlichen Hemmnissen sowie die Förderung der grenzüberschreitenden Erbringung von Dienstleistungen zum Ziel. Nach dreijähriger Übergangsphase sollte ihre Umsetzung bereits 2009 abgeschlossen sein. Doch bei der Liberalisierung des Dienstleistungsmarktes hakt es weiterhin. „Auch wenn man es sich aus nationaler Sicht manchmal nicht vorstellen kann, es gibt nichts, was Ewigkeitswert hat“, erläuterte die Referentin an Beispielen aus anderen Sektoren.

Was den Bereich Gesundheit anginge, gehe es darum, die Vorteile des deutschen Systems weiterzutragen und sich Bündnispartner auf europäischer Ebene für die Freiberuflichkeit zu suchen. „Es wird festzustellen sein, ob die Freiberuflichkeit in anderen Ländern etwas gilt. Und letztlich geht es darum, Mehrheiten herzustellen“, betonte Kalbfleisch-Kottsieper.

\section{Verbündete in Europa finden}

Die stellvertretende FVDZ-Bundesvorsitzende Dr. Gudrun Kaps-Richter bekräftigte, dass der Freie Verband in dieser Hin- sicht gut aufgestellt sei und flexibel auf die neuen Herausforderungen reagieren könne. „Wir suchen auch auf dieser Ebene nach neuen Ansätzen“, sagte Kaps-Richter und verwies auf die bereits bestehenden Partnerschaften mit anderen europäischen zahnärztlichen Verbänden. Die freien Berufe seien stark in die rechtlichen Rahmenbedingungen eingebunden - und die könnten in den einzelnen Nationalstaaten stark voneinander abweichen. „Deshalb ist es sinnvoll, erst einmal die Rahmenbedingungen schrittweise zusammenzuführen - und zu europäisieren “, sagte Kaps-Richter.

Ein großes Problem des zunehmenden Hineinwirkens Brüssels in die nationalen Gesundheitsbereiche sei, dass gesundheitspolitische Entscheidungen, die für andere Bereiche der Medizin getroffen würden, immer auch Auswirkungen auf die Zahnmedizin hätten. Kaps-Richter räumte ein, dass die europäische Dynamik sicherlich eine Zeitlang unterschätzt worden sei. Umso wichtiger sei es jetzt, zunächst auch national nach einem Weg für die Zahnärzte zu suchen, sich aus dem langen Schatten der Ärzteschaft in eine neue Form der Freiberuflichkeit freizuschwimmen, wie es der Freie Verband angekündigt hat. „Dafür werden wir uns in Europa Mitstreiter suchen“, sagte die stellvertretende FVDZ-Bundesvorsitzende.

Sabine Schmitt

\title{
Forum Zukunft in Berlin: Information, Interaktion, Kommunikation - Sicher in die Freiberuflichkeit
}

$\mathrm{E}_{\mathrm{s}}^{\mathrm{s}}$ s gibt viele gute Gründe für eine Reise nach Berlin - Ende September gibt es in diesem Jahr noch einen mehr: Der Freie Verband Deutscher Zahnärzte (FVDZ) lädt zu seinem ersten „Forum Zukunft“ in die deutsche Hauptstadt ein. Interaktiv und kommunikativ wird es bei dem zweitägigen Treffen am 30. September und 1. Oktober um die sichere Zukunftsplanung für junge Zahnärztinnen und Zahnärzte gehen. „Wir wollen den Teilnehmern einerseits ein prall gefülltes Informationspaket mitgeben und gleichzeitig viel Raum dafür lassen, persönliche Fragen im Austausch mit den Referenten und anderen Teilnehmern zu klären“, erläutert die stellvertretende FVDZ-Bundesvorsitzende Dr. Gudrun Kaps-Richter, das neue Format.

Das Forum Zukunft startet mit einer Führung durch den Bundesrat am Nachmittag des Freitags (30. September). Mit einem Impulsreferat zum Engagement in der Kollegenschaft wird Forum-Organisatorin Kaps-Richter für den ersten Austausch der Teilnehmer vor einem gemeinsamen Abendessen sorgen. Am Samstag, 1. Oktober, geht es dann ab 9 Uhr direkt in medias res der vier Themenschwerpunkte: Rechtsanwältin Melanie Neumann wird im Modul „Beruf und Familie“ über neue Berufsmodelle referieren und Gelegenheit bieten, über wichtige Fragen und Sorgen in Hinblick auf eine Praxisgründung oder das Angestellt-Bleiben zu sprechen. Rechtsanwalt Michael Lennartz widmet sich beim Thema „Angestellt oder Chef" dem Übergang vom Studium in den Beruf und dann in die Freiberuflichkeit, erläutert Vorteile und Nachteile und wird sicherlich mit den Teilnehmern über das Thema „Flexibilität versus Sicherheit" reden. Prozess- und Qualitätsmanager Sascha Kötter hat alles im Köcher, was die Teilnehmer zur finanziellen Sicherheit wissen müssen. Es geht nicht nur um die Frage, wie man nach einer Praxisgründung ruhig schlafen kann, sondern vor allem darum zu wissen, wofür man eigentlich morgens aufsteht. Rhetorik-Trainer Peter Edwin Brand wird in seinem Modul viel über das Thema „Erfolgreich kommunizieren“ vermitteln, im Mittelpunkt steht die Kommunikation mit Patienten, Chef und Mitarbeitern. Von den Teilnehmern selbst wird ebenfalls intensive Kommunikation gefragt sein: In Workshops arbeiten sie nach den jeweiligen Impulsvorträgen gemeinsam an den Themen weiter. Moderatoren werden die Teams bei der Erarbeitung ihrer Themengebiete unterstützen. Zum Abschluss stellen die Gruppen ihre Ergebnisse im Plenum vor.

Das richtige Ambiente für das Zukunftsforum bietet an dem Wochenende das moderne Design-Tagungshotel „nhow Berlin“. Die Teilnahmegebühr beträgt für FVDZ-Mitglieder 149 Euro, für Nicht-Mitglieder 199 Euro. Studierende können für 25 Euro (Mitglieder) oder 50 Euro (Nicht-Mitglieder) an der Veranstaltung teilnehmen. Übernachtungskosten sind nicht in den Kongressgebühren enthalten. Anmeldungen sind im Internet unter www.fvdz.de/forum-zukunft.html möglich.

Weitere Informationen zum Forum Zukunft oder zur Anreise gibt es bei Frauke Garstka in der FVDZ-Bundesgeschäftsstelle in Bonn unter der Telefonnummer 0228 / 855732 oder per Mail unter fg@fvdz.de. 